\title{
Assessment of SSR Marker Transfer from the Cultivated Strawberry to Diploid Strawberry Species: Functionality, Linkage Group Assignment, and Use in Diversity Analysis
}

\author{
Thomas M. Davis ${ }^{1}$ and Laura M. DiMeglio \\ University of New Hampshire, Department of Plant Biology, 104 Rudman Hall, Durham, NH 03824 \\ Ronghui Yang, Sarah M.N. Styan², and Kim S. Lewers \\ USDA-ARS Fruit Laboratory, 10300 Baltimore Avenue, BARC-West Building 010A, Beltsville, \\ $M D$ 20705-2350
}

\begin{abstract}
AdDitional InDEX WORDs. Fragaria, microsatellite, simple sequence repeat
Aвstract. The cultivated strawberry, Fragaria xananassa Duchesne ex Rozier, originated via hybridization between octoploids $F$. chiloensis (L.) Mill. and $F$. virginiana Mill. These three octoploid species are thought to share a putative genome composition of AAA'A'BBB'B'. Diploid $F$. vesca $\mathrm{L}$., is considered to have donated the A genome. Current attention to the development of a diploid model system for strawberry genomics warrants the assessment of simple sequence repeat (SSR) marker transferability between the octoploid and diploid species in Fragaria L. In the present study, 23 SSR primer pairs derived from $F$. xananassa 'Earliglow' by genomic library screening were evaluated for their utility in six diploid Fragaria species, including eight representatives of $F$. vesca, four of $F$. viridis Weston, and one each of $F$. nubicola (Hook. f.) Lindl. ex Lacaita, F. mandshurica Staudt, F. iinumae Makino, and F. nilgerrensis Schltdl. ex J. Gay. SSR primer pair functionality, as measured by amplification success rate $(=100 \%$ - failure rate) in each species, was ranked (from highest to lowest) as follows: F. vesca $(98.4 \%)>F$. iinumae $(93.8 \%)=$ F. nubicola $(93.8 \%)$ $>F$. mandshurica $(87.5 \%)>F$. nilgerrensis $(75 \%)>F$. viridis $(73.4 \%)$. The extent to which these octoploid-derived SSR primer pairs generated markers that could be added to the $F$. vesca linkage map also was assessed. Of the $13 F$. xananassa SSR markers that segregated codominantly in the $F$. vesca mapping population, 11 were assigned to linkage groups based upon close linkages to previously mapped loci. These markers were distributed over six of the seven $F$. vesca linkage groups, and can serve as anchor loci defining these six groups for purposes of comparative mapping between $F$. vesca and $F$. xananassa.
\end{abstract}

Simple sequence repeat (SSR) or microsatellite markers are well-suited for the purpose of comparative linkage mapping between diploid and octoploid strawberry (Fragaria spp.). SSRs are more likely to be transferable among populations than are anonymous markers such as the randomly amplified polymorphic DNA (RAPD) and amplification fragment length polymorphism (AFLP) markers employed in the first generation linkage maps of the diploid model species, $F$. vesca (Davis and Yu, 1997), and octoploid, cultivated strawberry, F. ×ananassa (Lerceteau-Kohler et al., 2003), respectively. The potential of SSRs for having multiple, codominant alleles is a feature of particular relevance to linkage mapping in an octoploid, in which a single SSR primer pair has the potential to detect as many as eight SSR variants in

\footnotetext{
Received for publication 14 Nov. 2005. Accepted for publication 22 Mar. 2006 The authors thank Kate Rappaport for help with PCR reactions and Tina Sphon for reaction analyses. Thanks also to Tad Sonstegard and to the Beltsville Area Research Center for managing and supporting the genotyping facility. The authors also thank M.E. Shields, Rebecca Grube, Chris Neefus, and John Hartung plus the anonymous reviewers for their helpful comments. This is Scientific Contribution 2284 from the New Hampshire Agricultural Experiment Station (NHAES). This research was supported in part by NHAES Project $\mathrm{H} 433$.

Mention of trade names or commercial products in this publication is solely for the purpose of providing specific information and does not imply recommendation or endorsement by the University of New Hampshire, or the U.S. Department of Agriculture.

1To whom reprint requests should be addressed; e-mail: tom.davis@unh.edu ${ }^{2}$ Current address: Pioneer Hi-Bred International, Inc. ADuPont Company, Waimea Research Center, P.O. Box 609, Waimea, HI 96796.
}

a single individual. Moreover, costly genomic library screening - the source of the initial wave of Fragaria SSRs (Ashley et al., 2003; Cipriani and Testolin, 2004; Hadonou et al., 2004; James et al., 2003; Nourse et al., 2002; Sargent et al., 2003) — is being supplanted by expressed sequence tag (EST) database mining as a means of discovering new SSR loci (Lewers et al., 2005).

The evident potential for SSR marker transferability between $F$. vesca and the commercially cultivated strawberry, $F$. ×ananassa (Cipriani and Testolin, 2004; Hadonou et al., 2004), is among the features that make $F$. vesca an attractive and appropriate diploid model species for strawberry genomics (Sargent et al., 2004). Highly self-fertile inbred lines and useful mutant forms of $F$. vesca are already available as genetic tools, due in part to the long history of cultivation of European $F$. vesca ssp. vesca L. forma semperflorens (Duchesne) Staudt (Darrow, 1966), the so-called 'Alpine', everbearing form of $F$. vesca. The first $F$. vesca linkage map was based on the $\mathrm{F}_{2}$ generation of a cross between $F$. vesca ssp. vesca $\mathrm{L}$. forma semperflorens cultivar Baron Solemacher and F. vesca ssp. americana (Porter) Staudt accession WC6 (Davis and Yu, 1997). 'Baron Solemacher' carries two independently inherited, recessive, single gene traits: runnerless $(r)$ and everbearing $(s)$ (Brown and Wareing, 1965).

ANCESTRY AND GENOME COMPOSITION OF $\boldsymbol{F}$. XANANASSA. The cultivated strawberry, $F$. $\times$ ananassa, is known to have originated via hybridization between octoploids $F$. chiloensis and $F$. virginiana (Darrow, 1966). R. Bringhurst and collaborators have proposed that the latter two species and $F$. xananassa share a common 
genome composition: expressed initially as AAA'A'BBBB (Senanayake and Bringhurst, 1967) and later revised to AAA'A'BBB'B' (Bringhurst, 1990). Based upon cytological evidence, Senanayake and Bringhurst (1967) inferred a correspondence between the $F$. vesca genome and the A genome of the octoploids. F. vesca has by far the widest geographic distribution of any strawberry species (Hancock, 1999), and is widely regarded as a likely genome donor to the octoploid species. Therefore, the potential for SSR marker transfer between the octoploid Fragaria species, F. vesca, and other Fragaria diploids is of significant interest.

In the present study, SSR primer pairs derived by screening a library derived from $F$. xananassa 'Earliglow' (Lewers et al., 2005; Nourse et al., 2002) were evaluated for their capacities to amplify products and detect polymorphisms in a diverse sampling of diploid Fragaria germplasm, including representatives of six species. The extent to which these octoploid-derived SSR primer pairs generated markers that could be added to the $F$. vesca linkage map (Davis and Yu, 1997) was also assessed.

\section{Materials and Methods}

SSR GENOTYPING. Twenty-three SSR primer pairs were identified by screening a genomic library of $F$. ×ananassa 'Earliglow', as described elsewhere (Lewers et al., 2005; Nourse et al., 2002). Primer sequences for the primer pairs with Agricultural Research Service Fruit Lab (ARSFL) numbers employed here are reported in Lewers et al. (2005). Genotyping of SSR PCR products was performed in the manner previously described (Lewers et al., 2005).

Primer Pair functionality ANd diversity analysis. Initially, 23 SSR primer pairs were screened for their ability to amplify products (as manifested by the presence of a chromatographic peak, or peaks) and detect polymorphisms among 16 diploid accessions (Table 1) representing six species. A subset of 16 primer pairs was then selected for closer scrutiny, providing 256 accession $\times$ primer pair combinations used for examination of germplasm diversity and primer pair functionality. The PCR amplification failure rate (percentage) for each species was calculated as the number of failed amplifications in that species $(\times 100)$, divided by the total number of amplifications attempted in that species, where the denominator equals the number of representatives from the species in question times 16 (the number of primer pairs evaluated). The potential usefulness of 16 of these SSR primer pairs for detecting genetic relationships was assessed by subjecting the respective data set to a phenetic analysis. A data matrix was constructed, scoring the presence of PCR product as 1 and its absence as 0 . The computer program MVSP (Multi-Variate Statistical Package, version 3.13; Kovach Computing Services, Pentraeth, U.K.) was used to create a similarity matrix based on Nei and Li's (1979) similarity coefficient and to construct a dendrogram using the unweighted pair group method with arithmetic mean (UPGMA) cluster analysis. Bootstrap support values were obtained from 1000 replicates using the program Treecon [version 1.3b for Windows (Van de Peer and De Wachter, 1994)].

The numbers of PCR product variants in three self-compatible species were compared to those in three self-incompatible species using a data subset chosen to minimize the number of missing data points. For this test, a square root transformation was applied to the data set, on the basis (Zar, 1999) that it consisted of counts (i.e., number of variants for each accession).

MAPPING. Twenty-two SSR primer pairs, including most of those used in the diversity analysis, were evaluated for their
Table 1. Diploid Fragaria germplasm used to assess the functionality of SSR primer pairs derived from genomic sequences of $F$. ×ananassa 'Earliglow'. The species, subspecies, and name or accession number are provided with the location from which each originated and the source from whom the plants or seeds were obtained.

\begin{tabular}{|c|c|c|}
\hline Germplasm identification & Site of origin & Source ${ }^{z}$ \\
\hline \multicolumn{3}{|l|}{ F. vesca ssp. vesca } \\
\hline 'Yellow Wonder' (YW) & European cultivar & Burpee \\
\hline \multicolumn{3}{|l|}{ F. vesca ssp. americana } \\
\hline 'Pawtuckaway' (PAWT) & $\begin{array}{l}\text { New Hampshire } \\
\text { (Mt. Pawtuckaway) }\end{array}$ & T. Davis \\
\hline \multicolumn{3}{|l|}{ F. vesca $(\text { ssp. bracteata })^{\mathrm{y}}$} \\
\hline DN1C & California (Del Norte Co.) & T. Davis \\
\hline HP6A & California (Humboldt Co.) & T. Davis \\
\hline $\mathrm{BC} 23$ & $\begin{array}{l}\text { British Columbia } \\
\text { (South of Prince George) }\end{array}$ & T. Davis \\
\hline BC29 & $\begin{array}{l}\text { British Columbia } \\
\text { (East of Prince George) }\end{array}$ & T. Davis \\
\hline $\mathrm{BC} 35$ & $\begin{array}{l}\text { British Columbia } \\
\text { (near McBride) }\end{array}$ & T. Davis \\
\hline GS2C & $\begin{array}{r}\text { British Columbia } \\
\text { (near McBride) }\end{array}$ & G. Staudt \\
\hline \multicolumn{3}{|l|}{ F. mandshurica } \\
\hline GS99 & Jakutsk, Russia & G. Staudt \\
\hline \multicolumn{3}{|l|}{ F. nubicola } \\
\hline CFRA 520 (PI 551851) & Pakistan & NCGR \\
\hline \multicolumn{3}{|l|}{ F. iinumae } \\
\hline CFRA 377 (PI 551751) & Japan & NCGR \\
\hline \multicolumn{3}{|l|}{ F. nilgerrensis } \\
\hline CFRA 1358 (PI 616672) & Yunnan, China & NCGR \\
\hline \multicolumn{3}{|l|}{ F. viridis } \\
\hline CFRA 333 (PI 551741) & Germany & NCGR \\
\hline CFRA 341 (PI 551742) & Germany & NCGR \\
\hline SIB3 & Siberia & UNH \\
\hline NOV3A & Siberia & UNH \\
\hline
\end{tabular}

${ }^{\mathrm{z} A c c e s s i o n s}$ with CFRA prefixes were obtained from the USDANational Clonal Germplasm Repository (NCGR) in Corvallis, Ore. F. vesca'Yellow Wonder' was initially purchased as seed from W. Atlee Burpee and Co., Warminster, Pa., and was subsequently seed propagated through natural self pollination at the University of New Hampshire. GS2C and GS99 were obtained as seed from Guenter Staudt, Merzhauzen, Germany. SIB3 and NOV3A were obtained as seed from, respectively, Garrett Crow (University of New Hampshire, Durham) and Ivan Krasnoborov (Central Siberian Botanical Garden, Novosibirsk, Russia). Accessions DN1C, HP6A, PAWT, BC23, and BC29 were collected from the wild as runner plants by T.M. Davis.

yThe accessions listed as $F$. vesca ssp. bracteata were collected within the range of this subspecies, as delineated by Staudt (1999), but have not been definitively typed.

ability to detect polymorphism between mapping parents 'Baron Solemacher' (BS) and WC6 (Davis and Yu, 1997). The BS and WC6 SSR data set was not added into the diversity analysis data set because the two data sets were generated in separate assays that may have produced alternate measures of "size" for the same SSR variant, or identical measures of size for similar but distinct variants.

Following the minimal mapping population concept of Joyeux et al. (1999), an informative subset of $35 \mathrm{~F}_{2}$ generation plants from the original 80-plant mapping population of Davis and $\mathrm{Yu}$ (1997) was manually selected to maximize the number of detected crossovers and thus facilitate linkage group assignment and 
rough approximation of map distance using a minimum number of genotyping assays. In the present study, genotypic data from the mapping population subset for all of 14 previously mapped codominant RAPD markers, the DNA sequence-based marker $A d h$ (alcohol dehydrogenase), and isozyme markers $S d h$ (shikimate dehydrogenase) and Pgi-2 (phosphoglucose isomerase-2) were used to construct seven framework linkage groups in the program Map Manager (Manly and Olson, 1999). The SSR genotyping data set for this population subset was then entered into a single "new chromosome" file and mapped to the framework linkage groups using the "Distribute" function. Linkage relationships within each linkage group were then refined using the "Ripple" function. Map distances were expressed as "Morgan" map units (i.e., as recombination percentage). Any linkages detected by Map Manager based upon recombination percentages of greater than $20 \%$ were subjected to confirmation or rejection on the basis of chi-squared contingency testing.

\section{Results}

SSR PRIMER PAIR FUNCTIONALITY AND DIVERSITY ANALYSIS. Of 23 SSR primer pairs evaluated on the 16 germplasm accessions, six primer pairs (ARSFL numbers 9, 15, 16, 20, 25, and 26) failed to give satisfactory results, either due to inconsistent amplification, or (for ARSFL_9 only) an excessive number of chromatographic peaks. One other primer pair, ARSFL_28, reproducibly amplified a product in only one accession, $F$. iinumae. No further data are presented for the aforementioned seven primer pairs.

The remaining 16 primer pairs evaluated on 16 germplasm accessions provided 256 accession $\times$ primer pair combinations used for examination of germplasm diversity and SSR primer pair functionality. Successful amplification of a PCR product is a necessary prerequisite to marker transfer. Among the 256 accession $\times$ primer pair combinations under consideration, 27 combinations reproducibly failed to produce a detectable chromatographic peak (Table 2). The failure rate in $F$. viridis, in which four accessions (CFRA 333, CFRA 341, NOV3A, and SIB3) were tested and 17 failed amplifications occurred, was $100 \times 17 /(4 \times 16)=26.6 \%$, the highest failure rate observed. At least one amplification failure occurred for 11 out of 16 primer pairs evaluated in $F$. viridis (Table 2). The comparable failure rates (in parentheses) for the other species tested, in descending order, were $F$. nilgerrensis (25\%), F. mandshurica (12.5\%), F. nubicola (6.2\%), F. iinumae (6.2\%), and $F$. vesca $(1.6 \%)$.

Germplasm DIVERSITY. The numbers and sizes of amplification products (detected as chromatographic peaks) generated from each accession with each of the 16 primer pairs under consideration are presented in Table 2 . Of the 256 accession $\times$ primer pair combinations assayed, 174 generated single peaks, 52 generated two peaks, and three generated three peaks (Table 2 ). When summed over all plant accessions, the number of SSR variants detected per primer pair ranged from six (ARSFL_19) to 19 (ARSFL_13), with a mean of 11.1. ARSFL_19 was the only primer pair that did not detect multiple variants in at least one accession, while at the opposite extreme, primer pair ARSFL_4 detected two variants in each of eight accessions.

The differences in the number of representatives per species and the non-random distribution of amplification failures among species present obstacles to interspecies comparisons of SSR PCR product diversity in the total data set. However, if $F$. viridis accession CFRA 341 is excluded from consideration because of its apparently low sequence similarity with 'Earliglow', the source of the SSR primer pairs, there were eight primer pairs (ARSFL numbers $4,7,10,17,19,22,24$, and 27) that amplified one or more products in each of the other 15 accessions. Considering only this defined data subset (shaded data, Table 2), and summing SSR PCR product diversity for each accession over the eight respective primer pairs, the total number of SSR PCR product size variants per accession ranged from a low of eight (the minimum possible for eight primer pairs) in $F$. vesca accessions GS2C, PAWT, and YW, to 13 in $F$. viridis accession NOV3A. When the known breeding system characteristics (Staudt, 1989) of the species are used to group the species, the mean number of variants per accession was 9.1 in the group of self-compatible (SC) species ( $F$. vesca, F. iinumae, and $F$. nilgerrensis), and 11.0 in the group of self-incompatible (SI) species ( $F$. viridis, $F$. nubicola, and $F$. mandshurica). The difference in mean number of variants between SC and SI species is statistically significant $(P=0.047)$ based on a two-tailed $t$ test.

Phenetic Analysis. The results of the cluster analyses are displayed graphically in Figure 1. Only four nodes in the UPGMA dendrogram have bootstrap support above $50 \%$. All of the $F$. vesca accessions, including seven North American accessions and one European accession (YW), form a cluster with 52\% bootstrap support. Within the $F$. vesca cluster, two of the four accessions from British Columbia (BC23 and BC29) form a subcluster with $51 \%$ bootstrap support; however, the other two accessions from British Columbia (BC35 and GS2C) fall just outside this subcluster. The four $F$. viridis accessions comprised another cluster, which has the highest bootstrap value (72\%) of any cluster in the tree.

SSR MAPPING IN $\boldsymbol{F}$. VESCA. Of 22 SSR primer pairs evaluated in the BS and WC6 mapping parents, 13 primer pairs detected single band mobility polymorphisms that distinguished the parents and that segregated as codominant markers in the informative mapping population subset. Identities of all evaluated primer pairs, and the allele sizes of polymorphic markers, are reported in Table 3. For the segregating markers, four phenotypic categories were recognized and encoded for linkage analysis: parental (BS), heterozygous, parental (WC6), and no band detected. The latter category was likely due to amplification failure for technical reasons: such reactions were not repeated. Because the informative mapping population subset was not a randomly chosen sample of the original mapping population (Davis and Yu, 1997), goodness-of-fit tests to a monohybrid expectation were not appropriate and were not performed.

Eleven of the SSR primer pairs detected single loci that were assigned to linkage groups on the basis of close $(<15 \%$ recombination frequency) linkages to previously mapped markers (Table 3). Three loci each were located on linkage groups II and III; two were located on group I; and one each was located on groups V, VI, and VII. No SSR marker was assigned to group IV. The three codominant SSR markers assigned to group II clustered into two unlinked subgroups, one of which was anchored by the previously mapped codominant markers Adh and BC103AB, and the other by previously mapped codominant marker Pgi-2 (Table 3). When the recessive linkage group II marker $r$ ( = runnerless) was included in the analysis, the two subgroups were loosely connected (results not shown), in agreement with the linkage relationships of markers Adh, BC103AB, $r$, and Pgi-2 in the original $F$. vesca map (Davis and Yu, 1997). SSR markers ARSFL_11 and ARSFL_15 were not linked to any of the previously mapped anchor loci, and so could not be assigned to any linkage group. 
Table 2. Size (base pairs) of amplification products from PCR reactions using 16 primer pairs derived from genomic sequences of Fragaria xananassa 'Earliglow', an octoploid strawberry cultivar, to assay 16 diploid strawberry germplasm accessions maintained at the University of New Hampshire. For each SSR primer pair, the total number of PCR size variants amplified from this collection of diploid germplasm representatives is summed in the far right column

Diploid strawberry germplasm accessions evaluated

names

\begin{tabular}{|c|c|c|c|c|c|c|c|c|c|c|c|c|c|c|c|c|c|}
\hline names & & & & & & & & & & & & & & & & & (no.) \\
\hline ARSFL_1 & $247^{z}$ & 247 & 264 & $\varnothing^{\mathrm{y}}$ & 254 & 283 & 250 & 263 & $\varnothing$ & $\varnothing$ & $\varnothing$ & 264 & $\begin{array}{l}240 \\
252\end{array}$ & 279 & 269 & $\varnothing$ & 10 \\
\hline ARSFL_2 & 238 & 275 & $\begin{array}{l}240 \\
246\end{array}$ & 242 & 279 & 281 & $\begin{array}{l}238 \\
256\end{array}$ & 282 & $\varnothing$ & 235 & 245 & 235 & $\varnothing$ & 245 & $\varnothing$ & 246 & 12 \\
\hline ARSFL_3 & $\begin{array}{l}318 \\
322\end{array}$ & 318 & $\begin{array}{l}318 \\
325\end{array}$ & 328 & 328 & 322 & $\begin{array}{l}312 \\
316\end{array}$ & 325 & $\varnothing$ & 311 & $\begin{array}{l}294 \\
312\end{array}$ & 311 & 314 & $\varnothing$ & 314 & $\varnothing$ & 9 \\
\hline ARSFL_4 $4^{x}$ & $\begin{array}{l}170 \\
174\end{array}$ & $\begin{array}{l}170 \\
174\end{array}$ & $\begin{array}{l}170 \\
174\end{array}$ & 174 & $\begin{array}{l}170 \\
186\end{array}$ & 170 & 174 & 196 & $\begin{array}{l}177 \\
197\end{array}$ & $\begin{array}{l}177 \\
197\end{array}$ & 205 & $\begin{array}{l}191 \\
197\end{array}$ & 172 & $\begin{array}{l}180 \\
184\end{array}$ & 240 & 172 & 12 \\
\hline ARSFL_7 & 218 & 218 & 216 & 216 & 216 & 218 & 216 & 216 & 213 & $\begin{array}{l}213 \\
222 \\
248\end{array}$ & 213 & $\begin{array}{l}206 \\
213\end{array}$ & 225 & $\begin{array}{l}219 \\
223\end{array}$ & 231 & $\begin{array}{l}253 \\
265\end{array}$ & 12 \\
\hline ARSFL_8 & $\begin{array}{l}302 \\
309\end{array}$ & $\begin{array}{l}302 \\
309\end{array}$ & 311 & 302 & 304 & 293 & 304 & 293 & $\varnothing$ & $\varnothing$ & 306 & $\varnothing$ & $\begin{array}{l}304 \\
310\end{array}$ & 302 & 304 & $\varnothing$ & 7 \\
\hline ARSFL_10 & 223 & $\begin{array}{l}223 \\
227\end{array}$ & 235 & 223 & 233 & 215 & 223 & 231 & 230 & $\begin{array}{l}239 \\
264\end{array}$ & $\begin{array}{l}231 \\
244\end{array}$ & 232 & 233 & $\begin{array}{l}227 \\
239\end{array}$ & 254 & 231 & 10 \\
\hline ARSFL_11 & 277 & $\begin{array}{l}261 \\
265\end{array}$ & 275 & 271 & 271 & 267 & $\begin{array}{l}247 \\
255\end{array}$ & 255 & 296 & $\varnothing$ & 306 & 308 & $\varnothing$ & $\begin{array}{l}271 \\
279\end{array}$ & $\begin{array}{l}241 \\
252\end{array}$ & $\begin{array}{l}252 \\
271\end{array}$ & 14 \\
\hline ARSFL_12 & 261 & 261 & $\begin{array}{l}259 \\
261\end{array}$ & 261 & $\begin{array}{l}261 \\
263\end{array}$ & 263 & 259 & 261 & 257 & 261 & $\varnothing$ & $\varnothing$ & $\begin{array}{l}259 \\
261\end{array}$ & 320 & $\begin{array}{l}177 \\
257\end{array}$ & $\begin{array}{l}218 \\
268\end{array}$ & 8 \\
\hline ARSFL_13 & 302 & $\begin{array}{l}206 \\
352\end{array}$ & 285 & 299 & 280 & 278 & $\begin{array}{l}211 \\
339\end{array}$ & 167 & $\varnothing$ & $\varnothing$ & 243 & 253 & 208 & 197 & $\begin{array}{l}167 \\
193\end{array}$ & $\begin{array}{l}271 \\
275\end{array}$ & 19 \\
\hline ARSFL_14 & 215 & 232 & 215 & 214 & 217 & 234 & 231 & $\varnothing$ & 279 & $\varnothing$ & 280 & $\begin{array}{l}247 \\
293\end{array}$ & 222 & $\begin{array}{l}231 \\
246\end{array}$ & 229 & $\varnothing$ & 13 \\
\hline ARSFL_17 & 216 & 218 & 216 & 216 & 218 & 218 & 216 & 220 & 218 & $\varnothing$ & $\begin{array}{l}213 \\
220\end{array}$ & 216 & 217 & 219 & 257 & 222 & 8 \\
\hline ARSFL_19 & 314 & 328 & 314 & 328 & 328 & 328 & 328 & 328 & 342 & $\varnothing$ & 342 & 342 & 330 & 326 & 342 & 341 & 6 \\
\hline ARSFL_22 & 195 & 205 & $\begin{array}{l}189 \\
193\end{array}$ & 191 & 197 & $\begin{array}{l}176 \\
212\end{array}$ & 168 & 270 & 173 & 197 & $\begin{array}{l}189 \\
197\end{array}$ & 189 & $\begin{array}{l}150 \\
154\end{array}$ & $\begin{array}{l}167 \\
204\end{array}$ & 263 & 254 & 17 \\
\hline ARSFL_24 & $\begin{array}{l}258 \\
264\end{array}$ & 256 & 256 & 272 & $\begin{array}{l}245 \\
282\end{array}$ & 258 & 244 & 286 & $\begin{array}{l}203 \\
219\end{array}$ & 203 & $\begin{array}{l}203 \\
219\end{array}$ & 203 & 250 & 242 & $\begin{array}{l}162 \\
242\end{array}$ & 238 & 15 \\
\hline ARSFL_27 & 162 & 162 & 162 & 162 & 162 & 162 & 162 & 162 & $\begin{array}{l}186 \\
193\end{array}$ & $\varnothing$ & $\begin{array}{l}197 \\
207\end{array}$ & 203 & 162 & 164 & 162 & 157 & 8 \\
\hline
\end{tabular}

$\begin{array}{lllllllllllllllll}\text { Total number of } & 20 & 21 & 21 & 15 & 19 & 17 & 21 & 15 & 14 & 12 & 20 & 17 & 18 & 21 & 20 & 16\end{array}$

PCR products

per accession

${ }^{2}$ Size of amplified PCR product in base pairs (bp).

$y \varnothing=$ No amplification with this primer pair $\times$ accession combination.

x Shaded boxes indicate primer pairs that amplified at least one product from every accession (excluding CFRA 341 from which few primer pairs could amplify a product, indicating little sequence similarity to 'Earliglow'). 


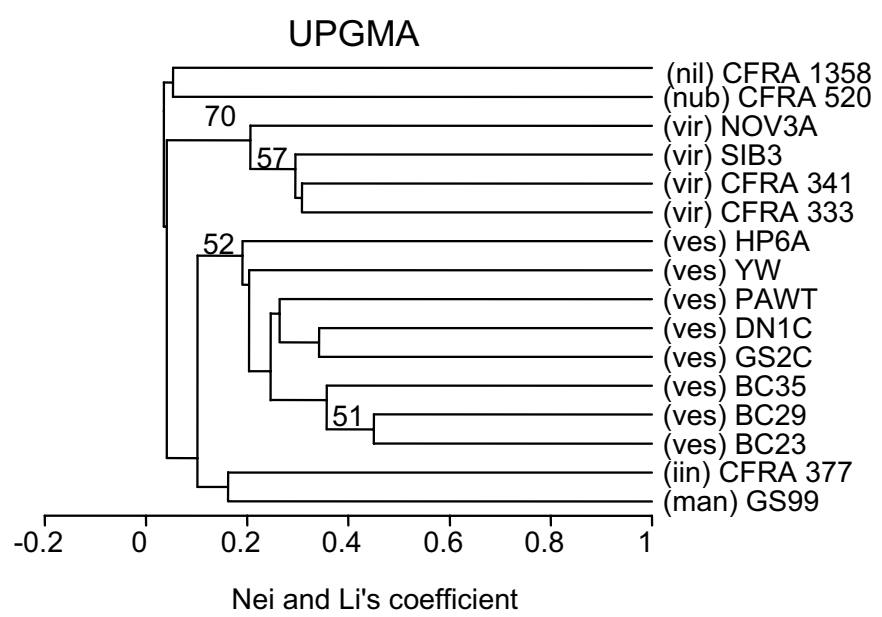

Fig. 1. Dendogram of Fragaria accessions representing six diploid species characterized with 16 SSR primer pairs. The six species are $F$. vesca (ves), $F$ viridis (vir), F. nubicola (nub), F. mandshurica (man), F. iinumae (iin), and $F$. nilgerrensis (nig). The SSR primer pairs were derived from genomic sequences of $F$. ×ananassa 'Earliglow'. The unrooted dendrogram was constructed using UPGMA cluster analysis of a similarity matrix generated using Nei and Li's coefficient. Bootstrap values over $50 \%$ are shown and were generated using UPGMA with 1000 replicates.

\section{Discussion}

Three criteria are applicable to the assessment of SSR marker transferability among taxa. First, an SSR primer pair based on DNA sequence from the source taxon or population must amplify a PCR product (i.e., must be functional) in one or more of the sampled members of the recipient taxon or population. Second, to be useful as a genetic marker in the recipient taxon or population, polymorphism must be detected between/among the population members of interest. Finally, marker codominance, as associated with band mobility polymorphism, is generally more useful than marker dominance, as associated with presence/absence polymorphism.

The transferability of SSR primer pairs among cultivars, populations, and subspecies within $F$. vesca has proven to be quite good (Cipriani and Testolin, 2004; Hadonou et al., 2004; James et al., 2003). When Hadonou et al. (2004) evaluated 31 primer pairs derived from $F$. vesca ssp. vesca on a sampling of diploid Fragaria germplasm that included all four $F$. vesca subspecies [vesca, americana, bracteata (A. Heller) Staudt, and californica (Cham. \& Schltdl.) Staudt], PCR amplifications were successful with all 15 of the $F$. vesca accessions, and 26 of the 31 primer pairs detected band mobility polymorphism in the sampled germplasm. When the latter 26 primer pairs were evaluated on $\mathrm{F}_{2}$ plants from a cross between $F$. vesca ssp. vesca $\mathrm{L}$. forma semperflorens cultivar Yellow Wonder x F. vesca ssp. americana 'Pawtuckaway', 20 of the primer pairs detected band mobility polymorphism and codominant marker segregation, while the remaining six markers were monomorphic in this intraspecific cross.

In comparison, when the interspecific cross $F$. vesca ssp. vesca FDP815 x F. nubicola FDP601 was used for map construction by Sargent et al. (2004), 14 out of 45 F. vesca-derived SSR primer pairs failed to amplify a product in the $F$. nubicola parent. In addition, one of five $F$. nubicola-derived and two of $12 F$. viridis-derived SSR primer pairs failed to amplify a product in the $F$. vesca parent. Both $F$. nubicola and $F$. viridis are diploids. Thus, the advantage of SSR marker codominance was partially sacrificed in this interspecific cross due to amplification failure for one or the other parental allele at each of 17 SSR loci. It was still possible to map the latter 17 loci as dominant markers, and 51 codominant SSRs also were mapped.

Table 3. Assignment of SSR markers derived from cultivated octoploid strawberry Fragaria $\times$ ananassa 'Earliglow' genomic sequences to diploid $F$. vesca linkage groups, as defined by linkages to markers (marked by asterisks) previously located on the $F$. vesca map (Davis and Yu, 1997). SSR primer pairs that failed to detect polymorphism between the mapping parents were: ARSFL numbers 7, 11, 16, 18, 19, 22, $23,26,28$.

\begin{tabular}{|c|c|c|c|c|}
\hline Linkage & SSR & Alle & (bp) & Closest linkages \\
\hline group & marker & $\mathrm{BS}$ & WC6 & (recombination \%) \\
\hline I & ARSFL_10 & 241 & 229 & 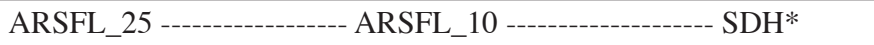 \\
\hline & & & & $(1.6)$ \\
\hline & ARSFL_25 & 187 & 181 & \\
\hline II & ARSFL_3 & 326 & 314 & $A d h^{*} / /$ ARSFL_12 ---------- ARSFL_3 --------- BC103AB* \\
\hline & & & & $(0.0)$ \\
\hline & ARSFL_12 & 261 & 258 & \\
\hline & & & & ARSFL_17 -------------'Pgi-2* \\
\hline & ARSFL_17 & 218 & 214 & $(3.0)$ \\
\hline III & ARSFL_8 & 291 & 302 & 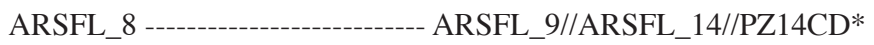 \\
\hline & & & & $\begin{array}{lll}(9.8) & (0.0) & (0.0)\end{array}$ \\
\hline & ARSFL_9 & 221 & 227 & \\
\hline & ARSFL_14 & 245 & 229 & \\
\hline $\mathrm{V}$ & ARSFL_1 & 261 & 244 & ARSFL_1 ---------------- PZ04CD* \\
\hline & & & & $(3.2)$ \\
\hline VI & ARSFL_2 & 280 & 235 & ARSFL_2 -------------- PX18AB* \\
\hline & & & & $(2.3)$ \\
\hline VII & ARSFL_4 & 194 & 174 & ARSFL_4 ----------------- BC194BD* \\
\hline & & & & $(3.1)$ \\
\hline
\end{tabular}


SSR marker transfer between $F$. viridis and $F$. vesca is also partially restricted. Sargent et al. (2003) developed 22 SSR primer pairs by screening a microsatellite-enriched $F$. viridis genome library, of which 21 primer pairs detected polymorphisms while one was monomorphic in a sampling of six $F$. viridis accessions. When these primer pairs were evaluated on $F$. vesca mapping parents 'Yellow Wonder' and 'Pawtuckaway', seven of $22 \mathrm{~F}$. viridis primer pairs (32\%) failed to consistently amplify a product in either parent (Sargent et al., 2003); thus, these F. viridis SSR loci were not transferable to the $F$. vesca mapping population. The remaining $15 F$. viridis primer pairs detected product length polymorphisms, and thus could be mapped in the $F$. vesca population as codominant markers.

SSR primer pair transferability from diploid to octoploid strawberry germplasm has been assessed in three studies. All 31 of the $F$. vesca-derived primer pairs described by Hadonou et al. (2004) amplified one or more products in three $F$. ×ananassa cultivars, although three of the primer pairs produced only smears or multiple products of larger than the expected size. Of 20 SSR primer pairs developed from $F$. vesca by Cipriani and Testolin (2004), 19 amplified single or multiple products in F. xananassa cultivar Queen Elisa, while 16 of the 20 amplified single or multiple products in an unidentified accession of octoploid $F$. chiloensis. Lewers et al. (2005) evaluated the functionality in octoploid Fragaria species of 10 SSRs developed from $F$. vesca genomic DNA sequences (James et al., 2003) and four developed from $F$. vesca EST sequences. Of the SSRs that amplified a product from any Fragaria template DNA, all amplified one or more products from $F$. virginiana, $F$. chiloensis, and 13 of 14 amplified products from $F$. xananassa. The observed high levels of shared SSR primer pair functionality among $F$. vesca and the octoploids $F$. xananassa and $F$. chiloensis is consistent with the hypothesis that $F$. vesca is a genome contributor to the octoploids, but leaves open the question of whether the shared sequences are specific to the particular subgenome in the octoploids that was putatively contributed by $F$. vesca.

Octoploid-derived SSR primer pair functionality in diploid species was reported by Lewers et al. (2005). Of the $62 \mathrm{~F}$. ×ananassa-designed SSR primer pairs that amplified a product from any of the genotypes tested, 58 amplified a product from at least one of the diploid strawberry species tested, and all four SSR primer pairs designed from $F$. virginiana amplified a product from a diploid strawberry. Functionality of octoploid-derived SSR primer pairs in other octoploid species also was examined. Of the $62 \mathrm{~F}$. xananassa-derived primer pairs, 57 amplified a product from $F$. chiloensis, 57 from $F$. virginiana, and 58 from other representatives of $F$. ×ananassa. All four SSR primer pairs designed from $F$. virginiana were functional in $F$. virginiana, $F$. chiloensis, and F. ×ananassa (Ashley et al., 2003; Lewers et al., 2005).

In the present study, SSR primer pairs based upon DNA sequence from octoploid $F$. $\times$ ananassa were evaluated in representatives of six diploid Fragaria species, including eight representatives of $F$. vesca, four of $F$. viridis, and one each of $F$. nubicola, $F$. mandshurica, F. iinumae, and F. nilgerrensis. Because of the sampling imbalance within species in this exploratory survey, care must be taken in drawing conclusions about functionality and polymorphism detection in the test taxa. Nevertheless, the results reported here, particularly when considered in conjunction with those of previous studies of Fragaria SSRs, provide useful insight into the patterns of SSR primer pair transferability among Fragaria species.

Fragaria $\times$ ananassa SSR primer pair functionality, as mea- sured by amplification success rate (= $100 \%$ - failure rate) in the test species, was ranked (from highest to lowest) as follows: $F$. vesca $(98.4 \%)>F$. iinumae $(93.8 \%)=F$. nubicola $(93.8 \%)>F$. mandshurica $(87.5 \%)>$ F. nilgerrensis $(75 \%)>$ F. viridis $(73.4 \%)$. Even the lowest of these amplification success percentages (to F. viridis) is quite good compared with the poor to zero rates of Fragaria SSR primer pair functionality in other Rosoideae genera, as reported by Lewers et al (2005). Nevertheless, the observed disparity in amplification failure rates between $F$. vesca $(1.6 \%)$ and $F$. viridis $(26.6 \%)$ is sufficient to prompt closer examination of the assertion (Lewers et al. 2005) that sequences from all Fragaria species are equally useful in designing SSR primers for any Fragaria species.

SSR primer pair transfer between $F$. vesca and $F$. ×ananassa has encountered little to no restriction. In two prior studies that examined a combined total of $51 \mathrm{~F}$. vesca-derived SSR primer pairs (Cipriani and Testolin, 2004; Hadonou et al., 2004), over $90 \%$ were functional in $F$. $\times$ ananassa. Similarly, the functionality in $F$. vesca of $F$. ×ananassa SSR primer pairs was virtually unrestricted $(98.4 \%)$ in the present study. In contrast, $F$. viridis SSR marker function in F. vesca was restricted to $68 \%$ (15 out of 22) of $F$. viridis primer pairs that consistently amplified a product in either parent (YW and PAWT) of an $F$. vesca mapping population (Sargent et al., 2003). In the present study, $F$. ×ananassa primer pair function in $F$. viridis was similarly restricted $(73.4 \%)$. The reverse marker function test, from $F$. viridis to $F$. ×ananassa, was not examined in the present study. However, the foregoing discussion justifies the expectation that $F$. viridis primer pair function might be restricted, as compared to $F$. vesca primer pair function, in $F$. xananassa.

The absence of strong bootstrap support for the phenetic dendrogram provides no justification for an attempt to draw phylogenetic inferences from this data set. Nevertheless, the dendrogram does contain aspects of interest, particularly when considered in comparison to a similar dendrogram of $F$. vesca accessions characterized with 26 SSR primer pairs designed from genomic sequences of $F$. vesca 'Ruegen' (Hadonou et al., 2004). In the latter study, excluding two $F$. vesca accessions that did not cluster as expected based upon their assigned subspecies, a major clade comprised of European $F$. vesca ssp. vesca accessions, including 'Yellow Wonder', was distinguished (with 60\% bootstrap support) from the second major clade comprised of North American subspecies americana, bracteata, and californica accessions. In the present study, 'Yellow Wonder' did not separate from the North American F. vesca accessions, perhaps because too few SSR primer pairs were used, or because the source of SSR primer sequences is $F$. xananassa instead of $F$. vesca. In both studies the North American $F$. vesca representatives fell into a common cluster, providing no encouragement for the use of SSR primer pairs to differentiate the three North American $F$. vesca subspecies: americana (represented by PAWT in the present study and two accessions in the prior study); bracteata (represented by one accession in the prior study and six tentatively classified accessions in the present study); and californica (represented by two accessions in the prior study). This lack of subspecies resolution was unexpected, because SSR primer pairs are commonly used to distinguish crop cultivars. The resolution of $F$. vesca from $F$. viridis in the present UPGMA tree does point to the potential for using SSR primer pairs to assess possible gene flow between $F$. viridis and $F$. vesca ssp. vesca, which are sympatric over much of their common range of western Europe to Lake Baikal (Staudt, 1989 ) and form natural unilateral ( $F$. vesca $\times$. viridis) hybrids 
that have been recognized as a unique taxon, Fragaria $\times$ bifera Duchesne (Staudt et al., 2003).

The mean number of SSR variants per accession was higher in the group of three self-incompatible (SI) species (11.0 variants per primer accession) than in the group of three self-compatible species (9.1 variants per accession). This outcome is not surprising, given the small sample size and the expectation of a higher level of heterozygosity in self-incompatible vs. self-compatible species. Nevertheless, we have refrained from assuming that variants detected by any given primer pair in a single individual are necessarily allelic. The alternate possibility - that two variants detected in any individual by a particular primer pair may result from monomorphic character states at two distinct, co-amplified loci-must be taken into account. The assumption of allelism must be validated by genetic data, such as a monogenic segregation pattern observed for putative alleles in the segregating progeny of a genetic cross.

Of the $13 F$. xananassa SSR markers that segregated as codominant markers in the $F$. vesca mapping population, 11 were assignable to linkage groups based upon close linkages to previously mapped anchor loci. These markers were distributed over six of the seven $F$. vesca linkage groups, and can serve as anchor loci defining these six groups for purposes of comparative mapping. These results contribute to the continuing development of the $F$. vesca map, and constitute a significant step toward defining the correspondences between linkage groups in $F$. vesca and their counterparts in F. xananassa.

It will now be of interest to determine whether the close linkages between/among SSR markers detected, respectively, on F. vesca linkage group I (ARSFL_10 and ARSFL_25), linkage group II (ARSFL_3 and ARSFL_12), and linkage group III (ARSFL_8, ARSFL_9, and ARFSL_14) are conserved in $F$. ×ananassa. Similarly, it will be of interest to determine whether the close linkage detected in $F$. vesca between SSR marker ARSFL_17 and the Pgi-2 isozyme locus is conserved in the octoploids. An answer to the latter question would provide an informative bridge between SSRs and isozymes as the latest and the first generations of molecular marker types in strawberry, because PGI (phosphoglucoisomerase) is the best characterized isozyme system in Fragaria, and its variants and inheritance patterns have been extensively characterized in the cultivated species (Arulsekar et al., 1981).

\section{Literature Cited}

Arulsekar, S., R.S. Bringhurst, and V. Voth. 1981. Inheritance of PGI and LAP isozymes in octoploid cultivated strawberries. J. Amer. Soc. Hort. Sci. 106:679-683.

Ashley, M.V., J.A. Wilk, S.M. Styan, K.J. Craft, K.L. Jones, K.A. Feldheim, K.S. Lewers, and T.L. Ashman. 2003. High variability and disomic segregation of microsatellites in the octoploid Fragaria virginiana Mill. (Rosaceae). Theor. Appl. Genet. 107:1201-1207.

Bringhurst, R.S. 1990. Cytogenetics and evolution in American Fragaria. HortScience 25:879-881.

Brown, T. and P.F. Wareing. 1965. The genetical control of the everbearing habit and three other characters of Fragaria vesca. Euphytica 14:97-112.
Cipriani, G. and R. Testolin. 2004. Isolation and characterization of microsatellite loci in Fragaria. Mol. Ecol. 4:366-368.

Darrow, G.M. 1966. The strawberry: History, breeding, and physiology. Holt, Rinehart and Winston, New York.

Davis, T.M. and H. Yu. 1997. A linkage map of the diploid strawberry, Fragaria vesca. J. Heredity 88:215-221.

Hadonou, A.M., D.J. Sargent, F. Wilson, C.M. James, and D.W. Simpson. 2004. Development of microsatellite markers in Fragaria, their use in genetic diversity analysis and their potential for genetic linkage mapping. Genome 47:429-438.

Hancock, J.F. 1999. Strawberries. CABI, New York.

James, C.M., F. Wilson, A.M. Hadonou, and K.R. Tobutt. 2003. Isolation and characterization of polymorphic microsatellites in diploid strawberry ( $F$. vesca L.) for mapping, diversity studies and clone identification. Mol. Ecol. Notes 3:171-173.

Joyeux, A., M.G. Fortin, R. Mayerhofer, and A.G. Good. 1999. Genetic mapping of plant disease resistance gene homologues using a minimal Brassica napus L. population. Genome 42:735-743.

Lerceteau-Kohler, E., G. Guerin, F. Laigret, and B. Denoyes-Rothan. 2003. Characterization of mixed disomic and polysomic inheritance in the octoploid strawberry (Fragaria $\times$ ananassa) using AFLP mapping. Theor. Appl. Genet. 107:619-628.

Lewers, K.S., S.M.N. Styan, S.C. Hokanson, and N.V. Bassil. 2005. Strawberry GenBank-derived and genomic simple sequence repeat (SSR) markers and their utility with strawberry, blackberry, and red and black raspberry. J. Amer. Soc. Hort. Sci. 130:102-115.

Manly, K.F. and J.M. Olson. 1999. Overview of QTL mapping software and introduction to map manager QT. Mammalian Genome 10:327-34.

Nei, M. and W.H. Li. 1979. Mathematical model for studying genetic variation in terms of restriction endonucleases. Proc. Natl. Acad. Sci. USA 76:5269-5273.

Nourse, S.M., E.W. Fickus, P.B. Cregan, and S.C. Hokanson. 2002. Development of simple sequence repeat (SSR) molecular markers in strawberry, p. 48-53. In: S.C. Hokanson and A.R. Jamieson (eds.). Strawberry research to 2001. ASHS Press, Alexandria, Va.

Sargent, D.J., T.M. Davis, K.R. Tobutt, M.J. Wilkinson, N.H. Battey, and D.W. Simpson. 2004. A genetic linkage map of microsatellite, gene-specific and morphological markers in diploid Fragaria. Theor. Appl. Genet. 109:1385-1391.

Sargent, D.J.,A.M.Hadonou, and D.W. Simpson. 2003. Development and characterization of polymorphic microsatellite markers from Fragaria viridis, a wild diploid strawberry. Mol. Ecol. Notes 3:550-552.

Senanayake, Y.D.A. and R.S. Bringhurst. 1967. Origin of Fragaria polyploids. I. Cytological analysis. Amer. J. Bot. 54:221-228.

Staudt, G. 1989. The species of Fragaria, their taxonomy and geographic distribution. Acta Hort. 265:23-33.

Staudt, G. 1999. Systematics and geographic distribution of the American strawberry species. Taxonomic studies in the genus Fragaria (Rosaceae: Potentilleae). University of California Publications in Botany, Vol. 81. University of California Press, Berkeley.

Staudt, G., L. DiMeglio, T.M. Davis, and P. Gerstberger. 2003. Fragaria ×bifera Duch.: Origin and taxonomy. Botanische Jahrbücher für Systematik 125:53-72.

Van de Peer, Y. and R. De Wachter. 1994. TREECON for Windows: A software package for the construction and drawing of evolutionary trees for the Microsoft Windows environment. Computer Applications Bioscience 10:569-570.

Zar, H.J. 1999. Biostatistical analysis, 4th ed. Prentice Hall. Upper Saddle River, N.J. 